\title{
ACUTE AORTIC DISSECTION - CASE DESCRIPTION
}

\author{
Gustaw Wójcikik ${ }^{1,2,3}$, Jolanta Piskorz ${ }^{4}$ \\ ${ }^{1}$ The Department of Diagnostic Imagining, The Zofia Tarnowska neé Zamoyska Regional Hospital in Tarnobrzeg \\ ${ }^{2}$ The Department of Balneotherapy, Chair of Rehabilitation, Physiotherapy and Balneotherapy, \\ Medical University of Lublin \\ ${ }^{3}$ The Department of Social and Humanistic Studies, State Higher Vocational School Memorial \\ of Prof. Stanislaw Tarnowski in Tarnobrzeg \\ ${ }^{4}$ The Department of Anaesthesiology and Intensive Care Medicine, \\ The Zofia Tarnowska neé Zamoyska Regional Hospital in Tarnobrzeg
}

Wójcik G., Piskorz J. (2015), Acute aortic dissection - case description. Health Problems of Civilization, 2 (9), p. 39-42.

Summary: Aortic dissection is a tearing in the inner wall of the aorta which spreads along the wall of the vessel. Acute aortic dissection is one of acute aortic syndromes, that is, sudden clinical conditions which are associated with abnormalities of the aortic wall and threaten patient's life. It is estimated that the number of aortic dissections in Poland amounts to around 1000 cases per year. The symptoms of aortic dissection are so equivocal that they may suggest other diseases of various organs in the thorax and abdominal cavity.

The described case of a 57-year-old man is an example of a complication of hypertension which, as a result of an isometrical physical effort, caused tearing of an inner membrane and forming of an aortic dissection of type I in the DeBakey classification. After performing medical imagining the patient with the diagnosis of aortic dissection, hematoma in the pseudoaneurysm cavity and renal infarction was submitted to emergency cardiac surgery. The diagnostics and selection of a place at cardiac surgery were carried out within an hour. However, the surgery did not take place, because the patient died due to a circulatory collapse.

Acute aortic dissection is subject to high mortality rate during the first hour since the onset of symptoms. Therefore, rapid recognition is crucial for the further prognosis of the patient.

Keywords: aortic dissection, life-threatening condition, acute renal failure, ischemic changes

\section{Introduction}

Aortic dissection is a tearing in the inner wall of the aorta which spreads along the wall of the vessel. As a result of a rupture of the tunica intima, walls are separated and two channels are created: a true lumen and a false lumen. Both channels communicate with each other in the place of a tear in tunica. The false lumen consists of a thin tunica adventitia and is very prone to rupture (Vural et al. 2001). Aortic dissection is one of the most dangerous complications of the hypertension as it is subject to high mortality rate. The symptoms of aortic dissection are so equivocal that they may suggest other diseases of various organs in the thorax and abdominal cavity.

Aortic dissection can manifest itself with a pain within the thorax and abdominal cavity. However, it happens that the only symptoms of the dissections are dyspnoea and elevated D-dimer (Suzuki et al. 2009). The process of dissection is so rapid that in case of treatment abandonment $50 \%$ of patients die within two days, while within 3 months after the dissection mortality increases up to $90 \%$.

Frequency of aortic dissection in the USA per number of inhabitants is estimated at 2-3,5/100 thousand/year (Divchev et al. 2014). In Poland it may correspond to 800-1400 cases per year. The aortic dissection is four times more common in men than women (Bickerstaff et al. 1982).

Nowadays there are two classifications of aortic dissection in place: Stanford classification and DeBakey classification. The Stanford classification includes two types: type A, in which the dissection occurs in the

Address for correspondence: Gustaw Wójcik, The Zofia Tarnowska neé Zamoyska Regional Hospital in Tarnobrzeg, Szpitalna 1, 39-400 Tarnobrzeg, phone: +48 508247 049, e-mail: gustaww@tlen.pl

Tables: 0 Figures: 2 References: 12 Full-text PDF www.hpc.edu.pl Copyright (C) Pope John Paul II State School of Higher Education in Biała Podlaska, Sidorska 95/97, 21-500 Biała Podlaska Indexation: Index Copernicus, AGRO, ProQuest, Polish Medical Bibliography, Polish Ministry of Science and Higher Education. This is an open-access article distributed under the terms of the Creative Common Attribution Non-commercial license (http://creativecommons.org/licenses/by-nc/3.0), which permits use, distribution and reproduction in any medium, provided the original works is properly cited, the use is non-commercial and is otherwise in compliance with the license. 
ascending aorta, and type B, which concerns the remaining parts of the aorta. The DeBakey system distinguishes 3 types of dissection. Type I covers the whole aorta, from the ascending part to its bifurcation into the common iliac arteries. Type II concerns only the ascending aorta while type III, in which the dissection starts just below the departure of a left subclavian artery (Vincentelli et al. 2011)

\section{Case description}

The 57-year-old patient came to emergency department with a severe pain in the thorax and epigastrium. Initially, it was a stabbing pain, but in the course of time it became less bothersome and more diffuse. The patient had a problem with specifying the location of the strongest pain. Once he pointed to the thorax, once to the epigastrium. He stated that the pain had started in the thoracic spine, between the scapulae and he associated it with lifting a heavy object. The patient had a history of spinal problems, therefore he linked the pain with degenerative disc disease. The patient was treated for a few years with hypertension and regularly took medications. At the time of hospital admission his blood pressure was 185/110 mm Hg. He received an antihypertensive treatment with angiotensin-converting inhibitors - zofenil plus $30 \mathrm{mg}$. In the laboratory tests cardiac markers were within the normal limits. There was an increase in C-reactive protein and reduction in the level of concentration of creatinine. Since the patient pointed to epigastrium as a current location of the pain, he was sent to abdominal ultrasonography. It revealed two flow channels in the not enlarged aorta, which indicated an aortic dissection. The aortic dissection was discovered below the departure of the renal arteries. Free fluid in the abdomen had not been found. Diagnostics was expanded to include a CT scan. The test protocol which was used covered the whole thoraic aorta, together with the abdominal part and its branching into subclavian arteries. The test revealed an extensive aortic dissection from the aortic valve, the ascending aorta, through the aortic arch, the entire descending aorta, up to the subclavian arteries. The hematoma was found above the diaphragm and in the abdominal aorta the dissection transferred into the left renal artery. It resulted in the impairment of blood flow to the left kidney with the characteristics of infarction.

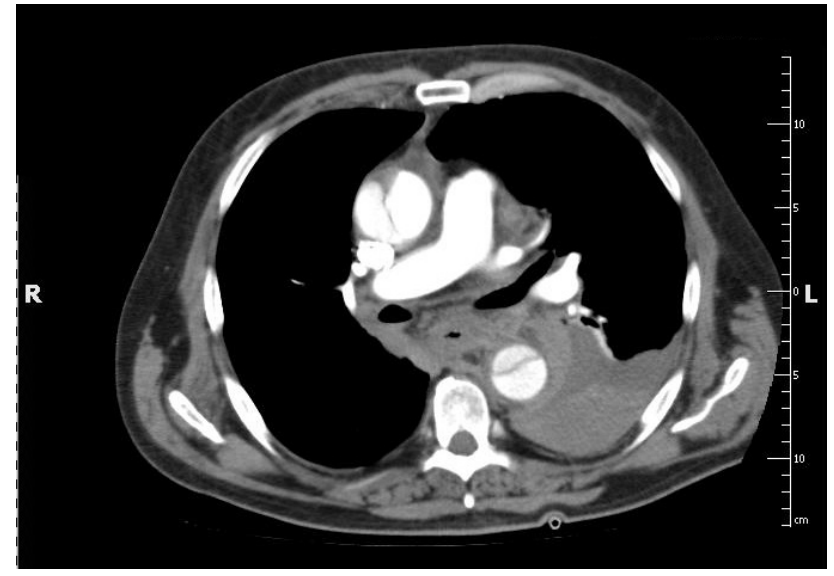

Figure 1. Angiography of a CT scan with visible aortic dissection of type I according to Stanford I

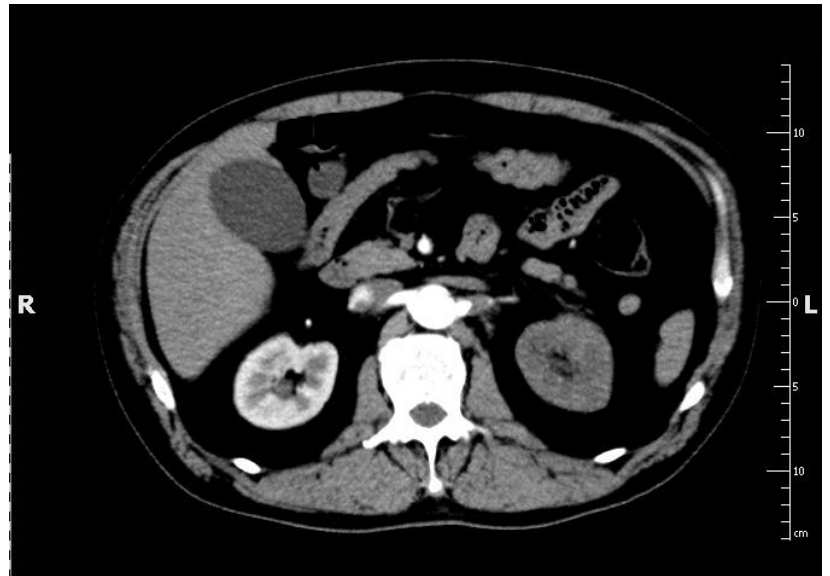

Figure 2. The creation of a false lumen caused the occlusion of the left renal artery and renal infarction

The hospital does not have the department of cardiac surgery so after the surgical consultation, the patient was immediately sent to a different hospital for treatment. The diagnostics and finding a place at cardiac surgery were carried out within an hour. However, the surgery did not take place, because the patient died during transport due to a circulatory collapse.

\section{Discussion}

Clinical symptoms of the aortic dissection are equivocal so in the differential diagnostics we should take into account musculoskeletal pain, a heart attack in progress, pulmonary embolism, mediastinal tumors, pleurisy, ascending cholangtis, peptic ulcer disease and acute pancreatitis (Galicia-Tornell et al. 2009). Acute aortic syndromes are characterized by interference in the integrity of the aortic walls which can have potentially devastating effects (Bonaca, O’Gara 2014).

Cardiovascular disease is the main cause of death in the world. Acute aortic syndromes proceed rapidly and can be hard to diagnose. New imaging techniques, such as transesophageal echokardiogram, CT scan and 
magnetic resonance imagining, allow for quick and accurate diagnosis of acute aortic syndromes. The rapid progress of the disease can make starting of a cardio surgical treatment impossible, even if the diagnostics was conducted efficiently. Cardio surgical treatment is necessary, especially in case of the dissection of the DeBakey's type I. Traditional classification systems, such as Stanford's or DeBakey's, facilitate deciding on early treatment by recognition of the high of death and serious complications connected with the occupation of the ascending aorta (Bonaca, O'Gara 2014).

In the case described here, the aortic dissection was caused by a substantial increase of blood pressure, which in connection with a physical effort caused rupture of a weakened wall of the aorta (Vural et al. 2001).

The described patient qualified for surgery due to a dissection from the aortic valve and hematoma in the pseudoaneurysm cavity. Conducting laboratory tests and imagining allowed for accurate diagnosis in less than an hour. However, due to lack of a cardiac surgery a transport to a hospital specializing in vascular injuries was immediately organized. Despite the effort of a rescue team, the patient did not survive. Unfortunately, a considerable distance $(70 \mathrm{~km})$ between the hospital which diagnosed the patient and the hospital where the surgery was to be performed was one of the main factors increasing death rate of the patient. The second factor was time which passed since the onset of pain symptoms to the moment when the patient reported to the emergency department. The patient said that the pain lasted for a few days. He was previously treated for a degenerative disc disease and he suspected a relapse. Therefore, he did not come to the hospital earlier, but waited for a spontaneous pain relief. Only after the pain had become unbearable and stopped to resemble radicular pain did he decide to seek medical help. However, time which passed since the onset of pain symptoms to the moment of diagnosis turned out to be decisive. Even if the hospital to which the patient came (with such a delay after the onset of symptoms) had had a cardiac surgery the chance of saving patient would have been higher but without warranty of recovery. Aortic dissection is a complication of a cardiac surgery with a high risk of mortality. Death rate in such cases is $25-50 \%$ (Williams et al. 2010).

Acute aortic dissection with the occlusion of renal arteries is not unusual. Frequent complication is also visceral ischaemia (Muraschita et al. 2012). Aortic dissection causes renal failure in 13,5\% of patients and in 9,2 $\%$ it may cause cerebral infarction (Williams et al. 2010).

There are also cases of aortic occlusion below level L1 which causes limb ischaemia and acute paraplegia (Galabada, Nazar 2014; Gargiulo et al. 2014).

Unfortunately, despite the use of improved diagnostic methods and surgical techniques, the death rate in case of aortic dissection is still very high (Fattori et al. 2008).

\section{References:}

1. Bonaca M.P., O'Gara P.T. (2014), Diagnosis and management of acute aortic syndromes: dissection, intramural hematoma, and penetrating aortic ulcer. Curr Cardiol Rep. 16(10): 536.

2. Divchev D., Aboukoura M., Weinrich M., Rehders T., Tillwich F., Richartz B., Clough R.E., Nienaber C.A. (2014), Risk evaluation of type B aortic dissection : Importance for treatment of acute aortic syndrome. Chirurg. 85(9): 774-81.

3. Galabada D.P., Nazar A.L. (2014), Unusual presentation of aortic dissection: Post-coital acute paraplegia with renal failure. Saudi J Kidney Dis Transpl. 25(5): 1059-61.

4. Fattori R., Tsai T.T., Myrmel T., Evangelista A., Cooper J.V., Trimarchi S., Li J., Lovato L., Kische S., Eagle K.A., Isselbacher E.M., Nienaber C.A. (2008), Complicated acute type B dissection: is surgery still the best option?: a report from the International Registry of Acute Aortic Dissection. JACC Cardiovasc Interv. 1(4): 395-402.

5. Galicia-Tornell M.M., Marín-Solís B., Mercado-Astorga O., Espinoza-Anguiano S., Martínez-Martínez M., Villalpando-Mendoza E. (2009), Sinus of Valsalva aneurysm with rupture. Case report and literature review. Cir Cir. 77(6): 441-5.

6. Gargiulo M., Bianchini Massoni C., Gallitto E., Freyrie A., Trimarchi S., Faggioli G., Stella A. (2014), Lower limb malperfusion in type B aortic dissection: a systematic review. Ann Cardiothorac Surg. 3(4): 351-67.

7. Murashita T., Ogino H., Matsuda H., Sasaki H., Tanaka H., Iba Y., Domae K., Fujiwara T. (2012), Clinical outcome of emergency surgery for complicated acute type B aortic dissection. Circ J. 76(3): 650-4.

8. Olsson C., Thelin S., Ståhle E., Ekbom A., Granath F. (2006), Thoracic aortic aneurysm and dissection: increasing prevalence and improved outcomes reported in a nationwide population-based study of more than 14,000 cases from 1987 to 2002. Circulation. 114(24): 2611-8.

9. Suzuki T., Distante A., Zizza A., Trimarchi S., Villani M., Salerno Uriarte J.A., De Luca Tupputi Schinosa L., Renzulli A., Sabino F., Nowak R., Birkhahn R., Hollander J.E., Counselman F., Vijayendran R., Bossone E., Eagle K. (2009), IRAD-Bio Investigators. Diagnosis of acute aortic dissection by D-dimer: the International Registry of Acute Aortic Dissection Substudy on Biomarkers (IRAD-Bio) experience. Circulation. 119(20): 2702-7. 
10. Williams M.L., Sheng S., Gammie J.S., Rankin J.S., Smith P.K., Hughes G.C. (2010), Aortic dissection as a complication of cardiac surgery: report from the Society of Thoracic Surgeons database. Ann Thorac Surg.; 90(6): 1812-7.

11. Vincentelli A., Juthier F., Banfi C., Prat A. (2011), Classification and etiology of acute aortic syndromes. Presse Med. 40(1): 28-33.

12. Vural K.M., Sener E., Taşdemir O., Bayazit K. (2001), Approach to sinus of Valsalva aneurysms: a review of 53 cases. Eur J Cardiothorac Surg. 20(1): 71-6.

Submitted: 12.02 .2015

Accepted: 29.04.2015 